\title{
Erratum to: Hemodynamic consequences of severe lactic acidosis in shock states: from bench to bedside
}

\author{
Antoine Kimmoun ${ }^{1,2,3}$, Emmanuel Novy ${ }^{1,2}$, Thomas Auchet ${ }^{1}$, Nicolas Ducrocq ${ }^{1}$ and Bruno Levy ${ }^{1,2,3^{*}}$
}

\section{Erratum}

Following publication of our article in Critical Care [1], the following error was brought to our attention. The sentence that reads "The rise in carbon dioxide partial pressure also increases hemoglobin affinity for oxygen and may, therefore, decrease oxygen delivery" is incorrect. The words "increases" and "decrease" were reversed.

The correct sentence should read: "The rise in carbon dioxide partial pressure also decreases hemoglobin affinity for oxygen and may, therefore, increase oxygen delivery." The original article also unfortunately published with the incorrect cover date. This was published with a cover date December 2015 whereas this should have been January 2016. This has been updated.

\section{Author details}

${ }^{1} \mathrm{CHU}$ Nancy, Service de Réanimation Médicale Brabois, Pole Cardiovasculaire et Réanimation Médicale, Hôpital de Brabois, Vandoeuvre-les-Nancy 54511, France. ${ }^{2}$ Université de Lorraine, Nancy 54000, France. ${ }^{3}$ INSERM U1116, Groupe Choc, Faculté de Médecine, Vandoeuvre-les-Nancy 54511, France.

Received: 8 February 2017 Accepted: 8 February 2017

Published online: 21 February 2017

\section{Reference}

1. Kimmoun A, Novy E, Auchet T, Ducroca N, Levy B. Hemodynamic consequences of severe lactic acidosis in shock states: from bench to bedside. Crit Care. 2015;19:175.

\footnotetext{
*Correspondence: b.levy@chu-nancy.fr

${ }^{1} \mathrm{CHU}$ Nancy, Service de Réanimation Médicale Brabois, Pole Cardiovasculaire et Réanimation Médicale, Hôpital de Brabois, Vandoeuvre-les-Nancy 54511, France

${ }^{2}$ Université de Lorraine, Nancy 54000, France
} 